Recebido em 11 de Junho de 2018 Aceito em 13 de Setembro de 2018 Autor para contato: raquelstefanello@yahoo.com.br

\title{
Germinação de sementes de Thymus vulgaris submetidas ao estresse salino
}

\author{
Germination of Thymus vulgaris seeds submitted to saline stress \\ Raquel Stefanello \\ Bruna Boucinha Viana \\ Luiz Augusto Salles das Neves \\ Universidade Federal de Santa Maria - UFSM - Santa Maria - Rio Grande do Sul - Brasil
}

\section{Resumo}

A alta concentração de sais no solo e na água é um dos fatores que limitam o crescimento e desenvolvimento das plantas em regiões com baixa disponibilidade hídrica. Neste contexto, o objetivo deste estudo foi avaliar a influência de diferentes concentrações de sais na germinação de sementes de tomilho. $\mathrm{O}$ experimento foi conduzido em câmara BOD, na temperatura constante de 20 oC e em presença de luz. As sementes foram colocadas sobre papel embebido em soluções aquosas de cloreto de cálcio $(\mathrm{CaCl} 2)$, cloreto de potássio $(\mathrm{KCl})$, cloreto de magnésio $(\mathrm{MgCl} 2)$ e cloreto de sódio ( $\mathrm{NaCl})$ nos potenciais osmóticos correspondentes a zero; -0,1; -0,2; -0,3 e -0,4 MPa. O efeito da salinidade foi avaliado através dos testes de germinação, primeira contagem e índice de velocidade de germinação. De acordo com os resultados é possível concluir que as sementes de tomilho toleram concentrações de $\mathrm{NaCl}$ até $-0,3 \mathrm{MPa}$ e $\mathrm{KCl}$ até $-0,2 \mathrm{MPa}$. Os sais $\mathrm{MgCl} 2$ e $\mathrm{CaCl} 2$ apresentam efeito negativo sobre a germinação e o vigor das sementes de tomilho a partir dos potenciais osmóticos de $0,1 \mathrm{MPa}$ e $-0,2 \mathrm{MPa}$, respectivamente.

\section{Abstract}

The high concentration of salts in soil and water is one of the factors limiting growth and development of plants in regions with low water availability. Within this context, the objective of this study was to evaluate the influence of different concentrations of salts on the germination of thyme

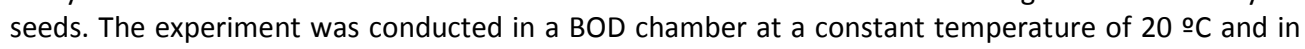
the presence of light. The seeds were placed on paper soaked with aqueous solutions of calcium chloride $(\mathrm{CaCl} 2)$, sodium chloride $(\mathrm{NaCl})$, potassium chloride $(\mathrm{KCl})$, and magnesium chloride $(\mathrm{MgCl} 2)$, at the osmotic potentials zero, $-0.1,-0.2,-0.3$, and $-0.4 \mathrm{MPa}$. The effect of the salinity was evaluated using a germination test, first count, and germination speed index. Based on the results, thyme seeds tolerate concentrations of $\mathrm{NaCl}$ to $-0.3 \mathrm{MPa}$ and $\mathrm{KCl}$ to $-0.2 \mathrm{MPa}$. The salts $\mathrm{MgCl} 2$ and $\mathrm{CaCl} 2 \mathrm{had}$ a negative effect on the germination and vigor of the thyme seeds for the osmotic potentials $-0.1 \mathrm{MPa}$ and $-0.2 \mathrm{MPa}$, respectively.

\section{Palavras-chave}

Processo germinativo. Salinidade. Tomilho.

\section{Keywords}

Germination process. Salinity. Thyme. 


\section{Introdução}

A salinidade é um dos principais fatores ambientais que diminui a produtividade das culturas e ameaça o equilíbrio alimentar global (Ashraf et al., 2015). Os efeitos do excesso de sais em sementes e plantas têm sido amplamente discutidos e podem causar limitações em todos os estádios de crescimento e desenvolvimento, comprometendo eventos fisiológicos que vão desde a germinação à produção (Secco et al., 2010). No entanto, os mecanismos de tolerância ao estresse salino em plantas e sementes ainda não estão suficientemente compreendidos, fazendo com que o progresso no desenvolvimento de culturas tolerantes à salinidade seja lento (Läuchli e Grattan, 2007).

Resultados da literatura mostram que a salinidade tem um efeito negativo sobre a percentagem de plântulas normais e emergência de várias culturas, tais como Guizotia abyssinica (Gordin et al., 2012); Petiveria alliacea (Lavezo et al., 2015); Hordeum vulgare (Askari et al., 2016); Salicornia europaea (Oslovsky et al., 2016); Phaseolus vulgaris (Khatar et al., 2017), Vigna sinensis (Kandil et al., 2017), Lens culinaris (Foti et al., 2018); Toona ciliata (Lucchese et al., 2018). De maneira geral, estes autores sugerem que os efeitos do excesso de sais em plantas estão ligados à redução do potencial osmótico do meio e aos efeitos tóxicos, causando diversos distúrbios metabólicos limitando a produtividade. No entanto, foram encontrados poucos estudos que avaliaram os efeitos de sais na germinação de sementes de tomilho (Cordovilla et al., 2014).

Os estudos relacionados com a resposta germinativa de sementes à condição de estresses artificiais têm importância para a agricultura e constituem-se em ferramentas que possibilitam a avaliação da taxa de sobrevivência e adaptação destas espécies às condições de estresses naturais (Guedes et al., 2013). Neste contexto, a semente de tomilho (Thymus vulgaris) foi escolhida para este estudo por apresentar poucos trabalhos científicos disponíveis relacionados ao desenvolvimento em condições de estresse salino e grande importância condimentar e medicinal. Esta espécie pertence à família Lamiaceae, é originária da região Mediterrânea Ocidental da Europa e produzida no sul e sudeste do Brasil. Possui uma forte fragrância herbácea e sua ação se dá em razão de excelentes propriedades broncopulmonares e antissépticas, sendo altamente eficaz em problemas das vias respiratórias. Seus principais componentes são fenóis, timol (40\%) e carvacrol (15\%) (Saqvic et al., 2007). O carvacrol tem sido investigado em decorrência de seu efeito bactericida, já o timol se destaca por seus efeitos antifúngicos e antibacterianos (Vieira de Melo et al., 2000). Seu cultivo não demanda muitas exigências, preferindo regiões secas, áridas, expostas ao sol e solos arenosos e calcários; é uma planta de solos pobres, rústica, devendo ser evitada a umidade e terras compactadas (Castro e Chemale, 1995).

Considerando um cenário amplo para entrada de sais nas lavouras brasileiras é de extrema importância elucidar os verdadeiros efeitos destes componentes nas culturas, contribuindo para novos conhecimentos nas áreas da biologia e agronomia auxiliando na adequada recomendação para o plantio nestas situações. Assim, objetivou-se com esse estudo avaliar a influência de diferentes concentrações de sais na germinação de sementes de tomilho.

\section{Material e métodos}

Para a avaliação do efeito tóxico dos sais no processo germinativo, as sementes de tomilho (Thymus vulgaris L.), adquiridas de uma empresa tradicional em produção e comercialização de sementes, foram semeadas em substrato papel embebido em solução aquosa de cloreto de cálcio $\left(\mathrm{CaCl}_{2}\right)$, cloreto de magnésio $\left(\mathrm{MgCl}_{2}\right)$, cloreto de sódio $(\mathrm{NaCl})$ e cloreto de potássio $(\mathrm{KCl})$. Os tratamentos receberam as seguintes denominações: $\mathrm{TO}=$ controle; $\mathrm{T} 1=-0,1 \mathrm{MPa} ; \mathrm{T} 2=-0,2 \mathrm{MPa} ; \mathrm{T} 3=-0,3 \mathrm{MPa} ; \mathrm{T} 4=-0,4 \mathrm{MPa}$. 0 nível zero 
correspondeu à testemunha, onde foi utilizada água destilada. A quantidade da solução necessária para obtenção dos potenciais osmóticos foi obtida pela equação de Van't Hoff citado por Taiz e Zeiger (2013) e por Braccini et al. (1996). O efeito tóxico dos sais no processo germinativo das sementes foi avaliado através dos seguintes testes:

Germinação (\%): realizado com quatro repetições de 100 sementes, distribuídas em caixas plásticas (gerbox), sobre três folhas de papel germitest umedecidas com água destilada ou solução salina na proporção de 2,5 vezes o peso do papel. Após a semeadura, as caixas plásticas foram mantidas em BOD, na temperatura constante de $20^{\circ} \mathrm{C}$, em presença de $8 \mathrm{~h}$ de luz e $16 \mathrm{~h}$ sem luz, sendo as contagens realizadas aos sete e 21 dias (quando foi finalizado o teste) e os resultados expressos em percentagem (Brasil, 2009).

Primeira contagem (\%): realizada juntamente com o teste de germinação, onde foi determinada a percentagem de plântulas normais no sétimo dia após a instalação do teste.

índice de velocidade de germinação (IVG): as contagens das sementes germinadas foram efetuadas diariamente, no mesmo horário utilizando-se como critério de germinação, a protrusão da raiz primária. $O$ índice de velocidade de germinação foi calculado empregando-se a fórmula de Maguire (1962).

Análise dos dados: o delineamento experimental utilizado foi o inteiramente casualizado, onde os tratamentos foram constituídos pelos diferentes sais e diferentes concentrações das soluções, com quatro repetições de 100 sementes. As variáveis percentagem de germinação e primeira contagem foram transformadas em arc sen $\sqrt{x / 100}$. Os dados foram submetidos à análise de variância pelo teste $F$ através do programa Sisvar (Ferreira, 2011).

\section{Resultados e discussões}

$\mathrm{Na}$ ausência de sais (controle), as sementes apresentaram, em média, germinação de $88 \%$, ocorrendo diferenças significativas em função dos tratamentos e dos sais utilizados (Fig. 1). Quando embebidas em solução de $\mathrm{MgCl}_{2} \mathrm{e} \mathrm{CaCl}_{2}$, não foi verificada germinação a partir dos potenciais -0,2 $\mathrm{MPa}$ e -0,3 $\mathrm{MPa}$, respectivamente. Além disso, na presença de $\mathrm{NaCl}$ ocorreu redução da percentagem de germinação a partir de $-0,1 \mathrm{MPa}(69 \%)$, enquanto em solução de $\mathrm{KCl}$ ocorreu decréscimo da germinação a partir de $-0,2 \mathrm{MPa}(18 \%)$ atingindo valores nulos no potencial de -0,4 MPa. É importante destacar que os efeitos dos sais $\mathrm{MgCl}_{2}$ e $\mathrm{CaCl}_{2}$ sobre a germinação das sementes foram mais acentuados que $\mathrm{NaCl}$ e $\mathrm{KCl}$. De acordo com Souza e Cardoso (2000), as diferenças químicas existentes entre estas soluções podem ocasionar alterações nos resultados de germinação e vigor das sementes, mesmo em potenciais hídricos similares. 

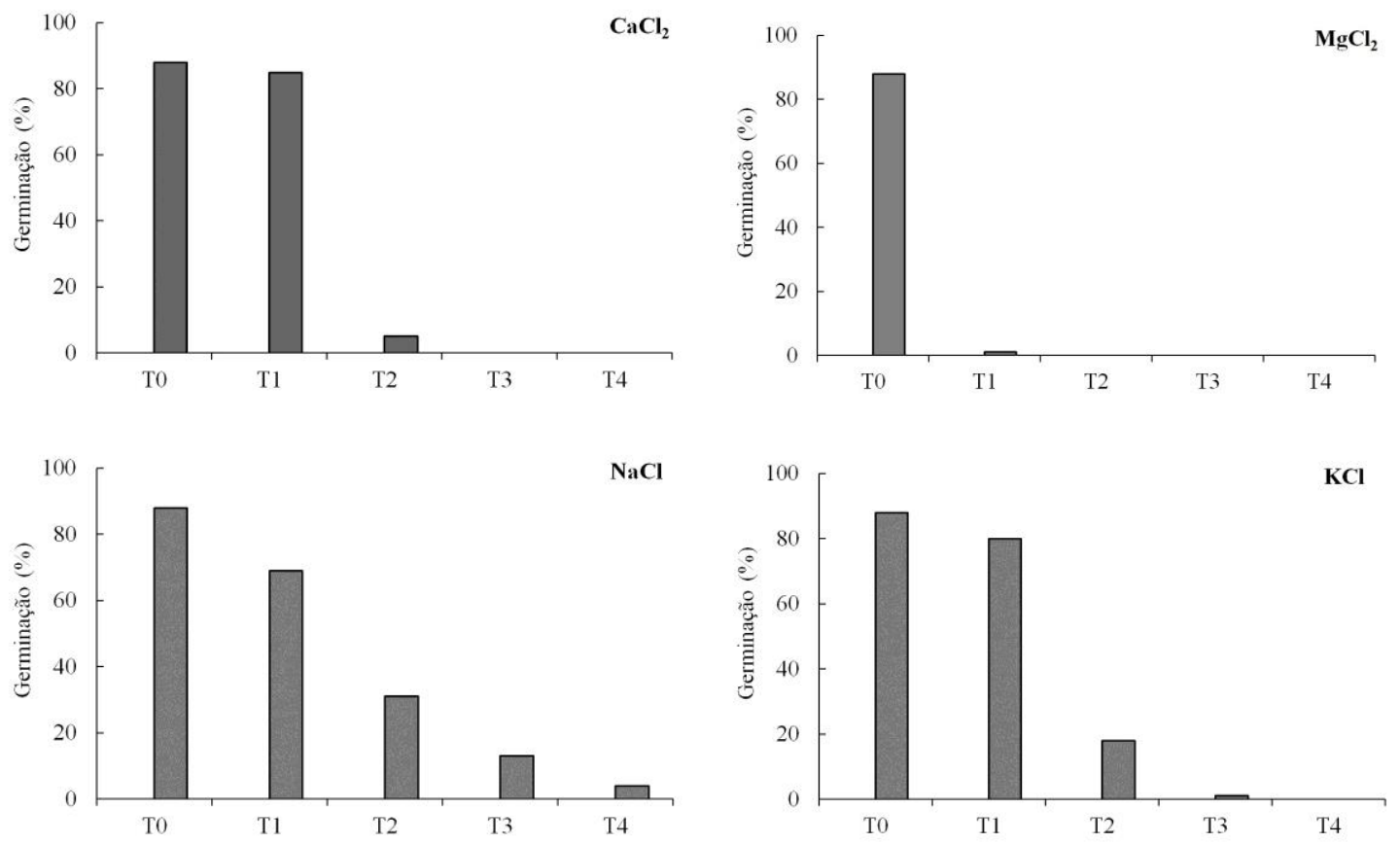

Figura 1. Percentagem de germinação de sementes de tomilho em diferentes concentrações de sais. Onde: $T 0=$ controle; $\mathrm{T} 1$ = -0,1 MPa; $\mathrm{T} 2$ = -0,2 $\mathrm{MPa} ; \mathrm{T} 3=-0,3 \mathrm{MPa} ; \mathrm{T} 4$ = -0,4 MPa.

Os resultados deste trabalho corroboram com estudos realizados por Oslovsky et al. (2016) onde a exposição a soluções salinas contendo $\mathrm{NaCl}, \mathrm{KCl}, \mathrm{CaCl}_{2}$ e $\mathrm{MgSO}_{4}$ reduziu a percentagem de germinação de sementes de Salicornia europaea. Além disso, Lucchese et al. (2018) observaram que a inibição total da germinação das sementes de Toona ciliata ocorreu a partir de -1,2 MPa de $\mathrm{NaCl}$. Da mesma forma, Askari et al. (2016) verificaram decréscimo da germinação de cevada com o incremento da concentração de $\mathrm{NaCl}$ e Lavezo et al. (2015) constataram que não ocorreu germinação das sementes de Petiveria alliacea, nos agentes $\mathrm{CaCl}_{2}$ e NaCl , nos potenciais osmóticos mais negativos que -0,4 e -0,5 MPa, respectivamente.

O estresse salino afeta substancialmente todas as características associadas à germinação e ao crescimento inicial de plântulas, com a tolerância à salinidade dependente da taxa de estresse nos genótipos selecionados (Foti et al., 2018). O efeito prejudicial da salinidade ocorre devido ao estresse osmótico e à toxicidade específica do íon. A interação de íons específicos e efeitos osmóticos leva a um decréscimo no número de sementes germinadas, bem como ao retardo na taxa de germinação (Kazemi e Eskandari, 2011). A redução do poder germinativo a partir das concentrações de $-0,1 \mathrm{MPa}\left(\mathrm{MgCl}_{2}\right),-0,2 \mathrm{MPa}(\mathrm{KCl}$ e $\mathrm{CaCl}_{2}$ ) e -0,3 MPa ( $\mathrm{NaCl}$ ), em comparação com o controle, serve como um indicador do índice de tolerância da espécie à salinidade. Sendo assim, a habilidade para germinar indica, também, a tolerância das plantas aos sais em estádios subsequentes do desenvolvimento (Taiz e Zeiger, 2013).

Em ambientes salinos, o crescimento das plantas é afetado e os efeitos prejudiciais incluem reduzida disponibilidade de água, devido a um efeito osmótico de altas concentrações de sais solúveis no meio da raiz; toxicidade iônica, como resultado do acúmulo de $\mathrm{Na}^{+}$e $\mathrm{Cl}^{-}$, estresse oxidativo, resultante de uma superprodução de espécies reativas de oxigênio (ROS) e deficiência aguda de $\mathrm{K}^{+}$como resultado do vazamento de $\mathrm{K}^{+}$de células despolarizadas (Shabala, 2013).

$O$ vigor das sementes, avaliado pelo teste de primeira contagem de germinação, foi reduzido com o aumento da concentração das soluções salinas (Fig. 2). Observou-se declínio da percentagem de plântulas normais a partir do potencial $-0,1 \mathrm{MPa}(\mathrm{NaCl}-51 \%)$ e $-0,2 \mathrm{MPa}$ 
( $\mathrm{KCl}$ - 10\%). Além disso, não foram observadas plântulas normais a partir do potencial -0,2 MPa quando utilizadas as soluções de $\mathrm{CaCl}_{2}$ e $\mathrm{MgCl}_{2}$.
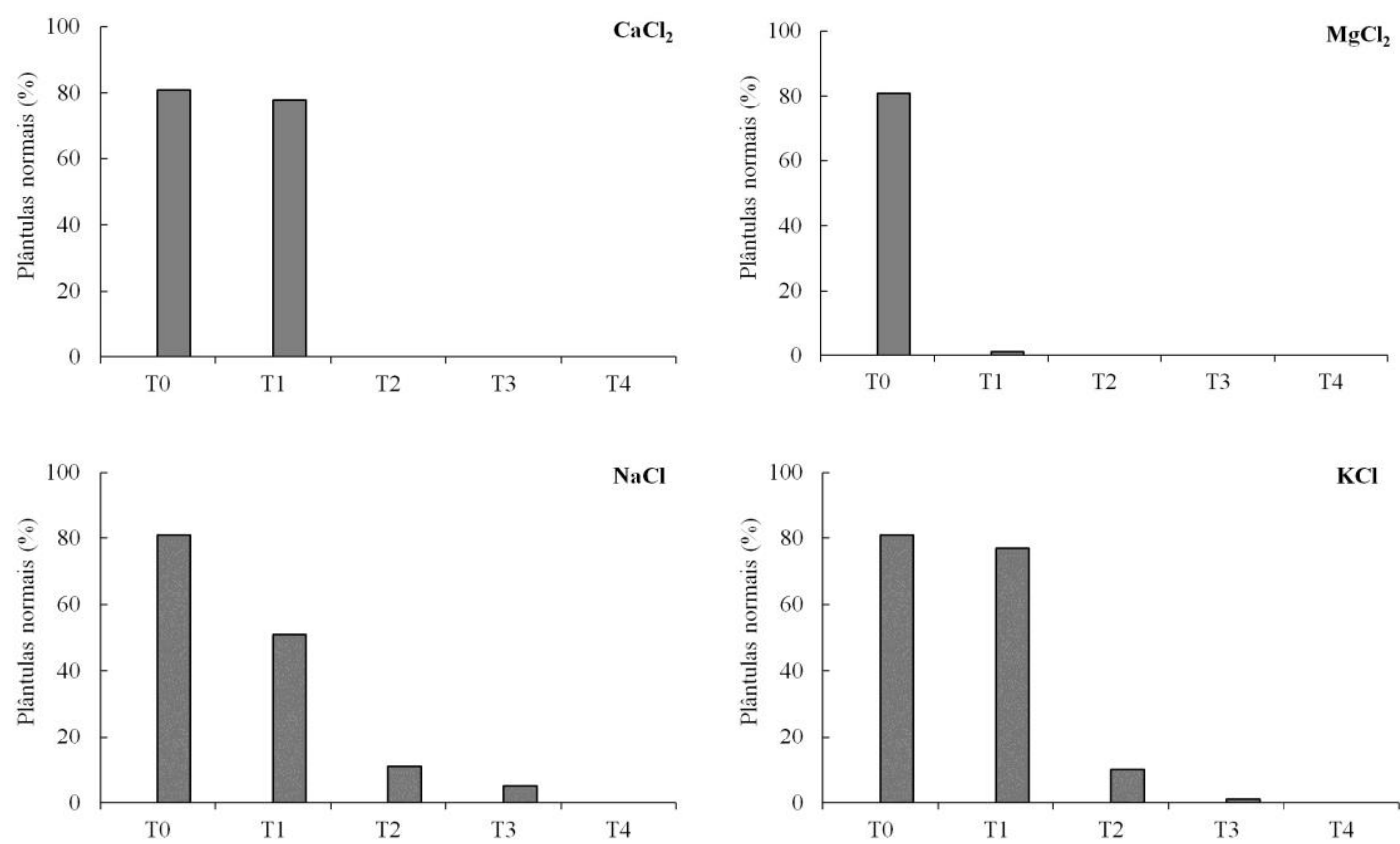

Figura 2. Primeira contagem de germinação de sementes de tomilho em diferentes concentrações de sais. Onde: T0 = controle; $\mathrm{T} 1$ = -0,1 MPa; $\mathrm{T} 2$ = -0,2 $\mathrm{MPa} ; \mathrm{T} 3$ = -0,3 $\mathrm{MPa} ; \mathrm{T} 4$ = -0,4 $\mathrm{MPa}$.

Esses resultados estão de acordo com os de outros estudos e fornecem evidências de que durante as fases do processo germinativo, a salinidade limita a absorção de água pela semente e, ao mesmo tempo, induz efeitos de toxicidade na germinação favorecendo o acúmulo de íons $\mathrm{Na}^{+}$e $\mathrm{Cl}^{-}$(Khajeh et al., 2003). Tais efeitos geralmente levam a uma redução na conversão enzimática de reservas armazenadas em açúcares, resultando assim na inibição da germinação, emergência incompleta de plântulas e subsequente mau estabelecimento de populações de plantas (Khan e Gulzar, 2003). Embora a salinidade afete drasticamente a germinação e o crescimento inicial, uma vez que a germinação ocorre nas camadas acima do solo, onde a maioria dos sais solúveis se acumulam, a severidade do seu efeito é fortemente dependente das espécies vegetais e, também, do seu genótipo (Almansouri et al., 2001).

Esta redução da porcentagem de plântulas normais durante o processo germinativo se deve ao fato de que a alta concentração de solutos em uma solução é um agente de estresse para as plantas, pois as soluções salinas promovem o déficit hídrico retendo água na solução de forma que o aumento da concentração a torna cada vez menos disponível para as plantas. Consequentemente, a alta concentração de sais presentes nas células pode inativar as enzimas, dificultar a síntese de proteínas e impedir a germinação de sementes (Taiz e Zeiger, 2013).

Em relação à velocidade de germinação, ocorreu a redução do IVG, conforme o estresse salino foi acentuado (Fig. 3). A velocidade de germinação foi reduzida a partir do potencial -0,1 $\mathrm{MPa}$ quando as sementes foram expostas aos sais $\mathrm{CaCl}_{2}$ e $\mathrm{MgCl}_{2}$. Por outro lado, na presença dos sais $\mathrm{KCl}$ e $\mathrm{NaCl}$ foram observados os menores índices a partir dos potenciais -0,2 MPa e $0,3 \mathrm{MPa}$ (respectivamente). 

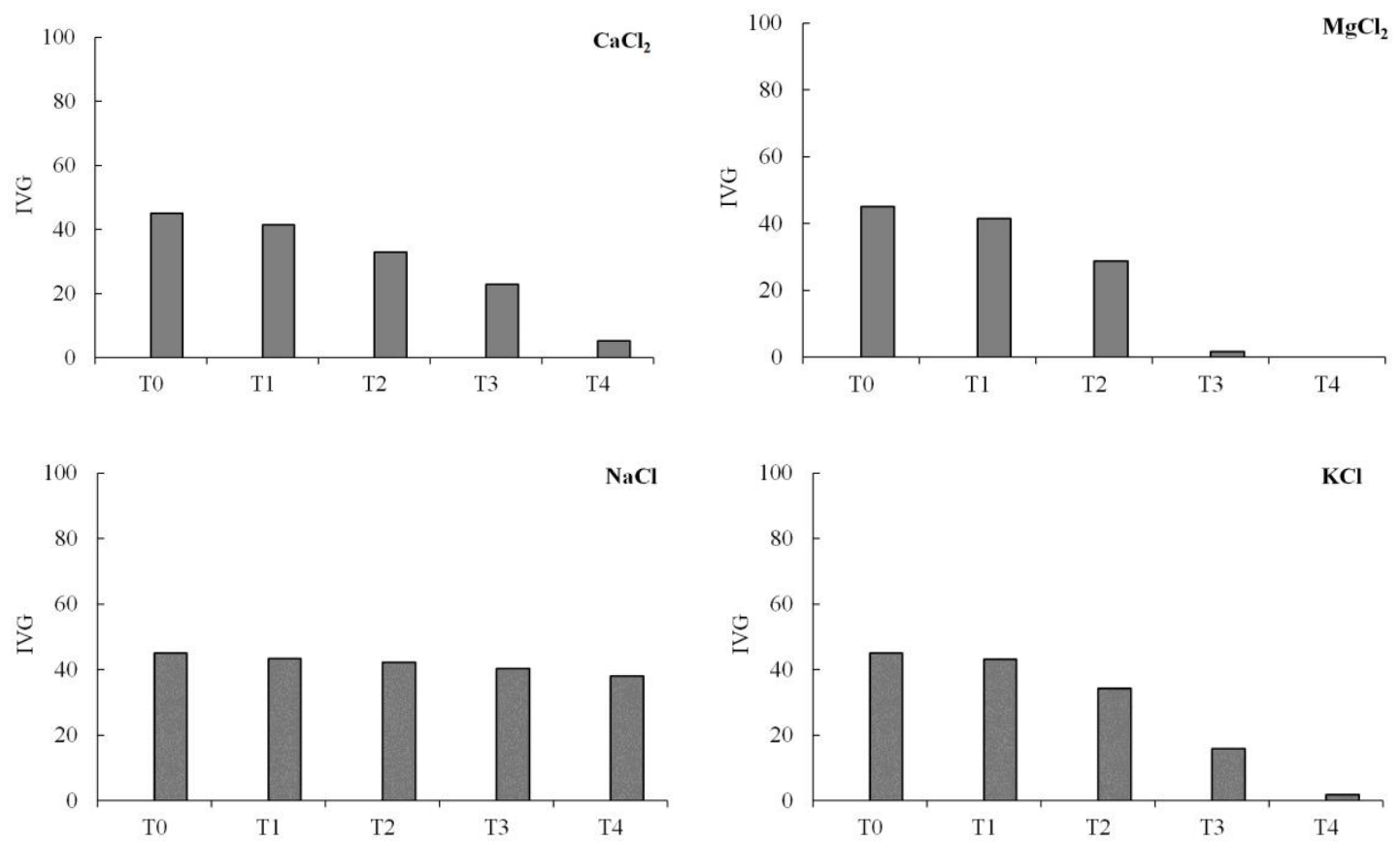

Figura 3. Índice de velocidade de germinação de sementes de tomilho em diferentes concentrações de sais. Onde: $\mathrm{T} 0$ = controle; $\mathrm{T} 1$ = -0,1 MPa; $\mathrm{T} 2=-0,2 \mathrm{MPa} ; \mathrm{T} 3$ = -0,3 MPa; $\mathrm{T} 4$ = -0,4 MPa.

Resultados equivalentes ao desta pesquisa foram obtidos por Gordin et al. (2012) onde verificaram redução gradativa da velocidade conforme a diminuição do potencial osmótico das soluções de $\mathrm{NaCl}$, evidenciando o efeito da salinidade no atraso da germinação de sementes de niger. De acordo com estes autores, a disponibilidade de água é um fator limitante para as fases iniciais do estabelecimento da planta e a redução do potencial hídrico do substrato resulta na diminuição da velocidade de germinação, sugerindo que nestas sementes os mecanismos de tolerância ao estresse salino não estejam presentes ou são ineficazes durante as fases iniciais de germinação.

Por fim, conclui-se que as sementes de tomilho mostraram-se sensíveis à exposição a diferentes sais nos potenciais osmóticos compreendidos entre -0,1 e -0,4 MPa, observado pelo decréscimo da germinação e do vigor. Estas informações são importantes para auxiliar no entendimento da distribuição geográfica da espécie, visto que nas regiões onde são cultivadas acabam expostas à adubação inadequada, irrigações mal conduzidas, menor frequência de chuvas decorrente de mudanças climáticas, bem como resíduos orgânicos presentes no solo onde as concentrações de sais podem atingir níveis elevados e, consequentemente, afetar o estabelecimento da cultura.

\section{Conclusões}

As sementes de tomilho toleram concentrações de $\mathrm{NaCl}$ até $-0,3 \mathrm{MPa}$ e $\mathrm{KCl}$ até $-0,2 \mathrm{MPa}$. Os sais $\mathrm{MgCl}_{2}$ e $\mathrm{CaCl}_{2}$ apresentam efeito negativo sobre a germinação e o vigor das sementes de tomilho a partir dos potenciais osmóticos de $-0,1 \mathrm{MPa}$ e $-0,2 \mathrm{MPa}$, respectivamente. 


\section{Referências}

1. ALMANSOURI, M.; KINET, J. M.; LUTTS, S. Effect of salt osmotic stresses on germination in durum wheat (Triticum durum Desf.). Plant and Soil, v. 231, n. 2, p. 243-254, 2001.

2. ASHRAF, A.; EL-MOHSEN, A. B. D.; EL-SHAFI, M. A.; GHEITH, E. M. S.; SULEIMAN, H. S. Using different statistical procedures for evaluation drought tolerance indices of bread wheat genotypes, Advance in Agriculture and Biology, v. 4, n. 1, p. 19-30, 2015.

3. ASKARI, H.; KAZEMITABAR, S. K.; ZARRINI, H. N.; SABERI, M. H. Salt tolerance assessment of barley (Hordeum vulgare L.) genotypes at germination stage by tolerance indices. Open Agriculture, v. 1, n.1, p. 37-44, 2016.

4. BRACCINI, A. L.; RUIZ, H. A.; BRACCINI, M. C. L.; REIS, M. S. Germinação e vigor de sementes de soja sob estresse hídrico induzido por soluções de cloreto de sódio, manitol e polietilenoglicol. Revista Brasileira de Sementes, v.18, n.1, p.10-16, 1996.

5. BRASIL. Ministério da Agricultura, Pecuária e Abastecimento. Regras para análise de sementes. Secretaria de Defesa Agropecuária. Brasília: Mapa/ACS, 2009. 399p.

6. CASTRO, L. O.; CHEMALE, V. M. Plantas medicinais: condimentares e aromáticas. Guairá: Agropecuária, 1995.194 p.

7. CORDOVILLA, M. P.; BUENO, M.; APARICIO, C.; URRESTARAZU, M. Effects of salinity and the interaction between Thymus vulgaris and Lavandula angustifolia on growth, ethylene production and essential oil contents. Journal of Plant Nutrition, v. 37, n. 6, p. 875-888, 2014.

8. FERREIRA, D. F. Sisvar: a computer statistical analysis system. Ciência e Agrotecnologia, v. 35, n. 6, p. 1039-1042, 2011.

9. FOTI, C.; KHAH, E. M.; PAVLI, O. I. Germination profiling of lentil genotypes subjected to salinity stress. Plant Biology, 2018.

10. GORDIN, C. R. B.; MARQUES, R. F., MASSETO, T. E.; SOUZA, L. C. F. Estresse salino na germinação de sementes e desenvolvimento de plântulas de niger (Guizotia abyssinica (Lf) Cass.). Acta Botanica Brasilica, v. 26, n. 4, p. 966-972, 2012.

11. GUEDES, R. S.; ALVES, E. U.; VIANA, J. S.; GONÇALVES, E. P.; LIMA, C. R.; SANTOS, S. R. N. Germinação e vigor de sementes de Apeiba tibourbou submetidas ao estresse hídrico e diferentes temperaturas. Ciência Florestal, v. 23, n. 1, p. 45-53, 2013.

12. KANDIL, A. A.; SHAREIF, A. E.; GAD, M. A. Effect of salinity on germination and seedling parameters of forage cowpea seed. Research Journal of Seed Science, v. 10, n. 1, p. 1726, 2017. 
13. KAZEMI, K.; ESKANDARI, H. Effects of salt stress on germination and early seedling growth of rice (Oryza sativa L.) cultivars in Iran. African Journal of Biotechnology, v. 10, n. 11, p. 17789-17792, 2011.

14. KHAJEH, H. M.; POWELL, A. A.; BINGHAM, I. J. The interaction between salinity stress and seed vigor during germination of soya bean seeds. Seed Science Technology, v. 31, n. 3, p. 715-725, 2003.

15. KHAN, M. A.; GULZAR, S. Germination responses of Sporobolus ioclados: a saline desert grass. Journal of Arid Environments, v. 53, n. 3, p. 387-394, 2003.

16. KHATAR, M.; MOHAMMADI, M. H.; SHEKARI, F. Some physiological responses of wheat and bean to soil salinity at low matric suctions. International Agrophysics, v. 31, n. 1, p. 83-91, 2017.

17. LÄUCHLI, A.; GRATTAN, S. R. Plant growth and development under salinity stress. In: JENKS, M. A.; HASEGAWA, P. M.; MOHAN, J. S. (Eds.), Advances in Molecular Breeding Towards Drought and Salt Tolerant Crops. p. 1-32, 2007.

18. LAVEZO, A.; BRAGA, L. F.; BATISTAO, A. C.; BONFANTE, L. V. Estresse osmótico na germinação de sementes de Petiveria alliacea L. Revista Brasileira de Plantas Medicinais, v. 17, n. 4, p. 622-630, 2015.

19. LUCCHESE, J. R.; BOVOLINI, M. P.; HILGERT, M. A.; BROSE, C. B.; AVRELLA, E. D.; LAZAROTTO, M. Estresse salino e hídrico na germinação e crescimento inicial de plântulas de Toona ciliata M. Roem. var. australis. Ciência Florestal, v. 28, n. 1, p. 141149, 2018.

20. MAGUIRE, J. D. Speed of germination and in selection and evaluation for seedlings emergence and vigor. Crop Science, v. 2, p. 176-177, 1962.

21. ORLOVSKY, N.; JAPAKOVA. U.; ZHANG, H.; VOLIS, S. Effect of salinity on seed germination, growth and ion content in dimorphic seeds of Salicornia europaea L. (Chenopodiaceae). Plant Diversity, v. 38, n. 4, p. 183-189, 2016.

22. SAQVIC, K. M.; KOSALEC, I.; MASTELIĆ, J.; PIECKOVÁ, E.; PEPELJNAK, S. Antifungal activity of thyme (Thymus vulgaris L.) essential oil and thymol against moulds from damp dwellings. Letters in Applied Microbiology, v. 44, n. 1, p. 36-42, 2007.

23. SECCO, L. B.; QUEIROZ, S. O.; DANTAS, B. F.; SOUZA, Y. A.; SILVA, P. P. Germinação de sementes de melão (Cucumis melo L.) em condições de estresse salino. Revista Verde de Agroecologia e Desenvolvimento Sustentável, v. 4, n. 4, p. 129-135. 2010.

24. SHABALA, S. Learning from halophytes: Physiological basis and strategies to improve abiotic stress tolerance in crops. Annals of Botany, v. 112, n. 7, p. 1209-1221, 2013.

25. SOUZA, G. M.; CARDOSO, V. J. M. Effects of different environmental stress on seed germination. Seed Science and Technology, v. 28, n. 3, p. 621-630, 2000. 
26. VIEIRA DE MELO, S. A. B.; COSTA, G. M. N.; GARAU, R.; CASULA, A.; PITTAU, B. Supercritical $\mathrm{CO}_{2}$ extraction of essential oils from Thymus vulgaris Linn. Brazilian Journal of Chemical Engineering, v. 17, n. 3, 2000.

27. TAIZ, L.; ZEIGER, E. Fisiologia vegetal. 5 ed. Porto Alegre: Artimed. 2013. 954p. 